Kragujevac Journal of Mathematics

Volume 42(1) (2018), Pages 29-39.

\title{
STEINER HARARY INDEX
}

\author{
YAPING MAO
}

\begin{abstract}
The Harary index $H(G)$ of a connected graphs $G$ is defined as $H(G)=$ $\sum_{u, v \in V(G)} \frac{1}{d_{G}(u, v)}$ where $d_{G}(u, v)$ is the distance between vertices $u$ and $v$ of $G$. The Steiner distance in a graph, introduced by Chartrand et al. in 1989, is a natural generalization of the concept of classical graph distance. For a connected graph $G$ of order at least 2 and $S \subseteq V(G)$, the Steiner distance $d_{G}(S)$ of the vertices of $S$ is the minimum size of all connected subgraphs whose vertex set contain $S$. Recently, Furtula, Gutman, and Katanić introduced the concept of Steiner Harary index and give its chemical applications. The $k$-center Steiner Harary index $S H_{k}(G)$ of $G$ is defined by $S H_{k}(G)=\sum_{S \subseteq V(G),|S|=k} \frac{1}{d_{G}(S)}$. Expressions for $S H_{k}$ for some special graphs are obtained. We also give sharp upper and lower bounds of $S H_{k}$ of a connected graph, and establish some of its properties in the case of trees.
\end{abstract}

\section{INTRODUCTION}

All graphs in this paper are undirected, finite, and simple. We refer to [3] for graph theoretical notation and terminology not described here. For a graph $G$, let $V(G), E(G)$, and $e(G)$ denote the set of vertices, the set of edges, and the size of $G$, respectively. Distance is one of the basic concepts of graph theory [4]. If $G$ is a connected graph and $u, v \in V(G)$, then the distance $d(u, v)=d_{G}(u, v)$ between $u$ and $v$ is the length of a shortest path connecting $u$ and $v$. If $v$ is a vertex of a connected graph $G$, then the eccentricity $\varepsilon(v)$ of $v$ is defined by $\varepsilon(v)=\max \{d(u, v) \mid u \in$ $V(G)\}$. Furthermore, the radius $\operatorname{rad}(G)$ and diameter $\operatorname{diam}(G)$ of $G$ are defined by $\operatorname{rad}(G)=\min \{\varepsilon(v) \mid v \in V(G)\}$ and $\operatorname{diam}(G)=\max \{\varepsilon(v) \mid v \in V(G)\}$. These latter two concepts are related by the inequalities $\operatorname{rad}(G) \leq \operatorname{diam}(G) \leq 2 \operatorname{rad}(G)$. Goddard and Oellermann gave a survey paper on this subject [13].

Key words and phrases. Distance, Steiner distance, Harary index, Steiner Harary $k$-index. 2010 Mathematics Subject Classification. Primary: 05C12. Secondary: 05C05; 05C35.

Received: July 18, 2016.

Accepted: November 6, 2016. 
The Wiener index $W(G)$ of $G$ is defined by

$$
W(G)=\sum_{u, v \in V(G)} d_{G}(u, v) .
$$

The first investigations of this distance-based graph invariant were done by Harold Wiener in 1947, who realized that there exist correlations between the boiling points of paraffins and their molecular structure, see [26-28]. Mathematicians study the Wiener index since the 1970s [10].

The Wiener index obtained wide attention and numerous results have been worked out, see the surveys $[9,15,16,30]$. The Harary index $H(G)$ of $G$ is defined by

$$
H(G)=\sum_{u, v \in V(G)} \frac{1}{d_{G}(u, v)} .
$$

For more details on the Harary index, we refer to [2, 17, 20, 29].

The Steiner distance of a graph, introduced by Chartrand et al. in 1989, is a natural and nice generalization of the concept of classical graph distance. For a graph $G(V, E)$ and a set $S \subseteq V(G)$ of at least two vertices, an $S$-Steiner tree or a Steiner tree connecting $S$ (or simply, an $S$-tree) is a subgraph $T\left(V^{\prime}, E^{\prime}\right)$ of $G$ that is a tree with $S \subseteq V^{\prime}$. Let $G$ be a connected graph of order at least 2 and let $S$ be a nonempty set of vertices of $G$. Then the Steiner distance $d_{G}(S)$ among the vertices of $S$ (or simply the distance of $S$ ) is the minimum size of connected subgraphs whose vertex set contain $S$. Note that if $H$ is a connected subgraph of $G$ such that $S \subseteq V(H)$ and $|E(H)|=d_{G}(S)$, then $H$ is a tree. Clearly, $d_{G}(S)=\min \{|E(T)|, S \subseteq V(T)\}$, where $T$ is subtree of $G$. Furthermore, if $S=\{u, v\}$, then $d_{G}(S)=d_{G}(u, v)$ is nothing new, but the classical distance between $u$ and $v$.

Let $n$ and $k$ be integers such that $2 \leq k \leq n$. The Steiner $k$-eccentricity $\varepsilon_{k}(v)$ of a vertex $v$ of $G$ is defined by $\varepsilon_{k}(v)=\max \left\{d_{G}(S)|S \subseteq V(G)| S \mid,=k\right.$, and $\left.v \in S\right\}$. The Steiner $k$-radius of $G$ is $s \operatorname{rad}_{k}(G)=\min \left\{\varepsilon_{k}(v) \mid v \in V(G)\right\}$, while the Steiner $k$ diameter of $G$ is $s \operatorname{diam}_{k}(G)=\max \left\{\varepsilon_{k}(v) \mid v \in V(G)\right\}$. Note that for every connected graph $G, \varepsilon_{2}(v)=\varepsilon(v)$ for all vertices $v$ of $G, s \operatorname{rad}_{2}(G)=\operatorname{rad}(G)$ and $s \operatorname{diam}_{2}(G)=$ $\operatorname{diam}(G)$. For more details on Steiner distance, we refer to $[1,5,6,8,13,25]$.

The following observation is easily seen.

Proposition 1.1. Let $k$ be an integer such that $2 \leq k \leq n$. If $H$ is a spanning subgraph of $G$, then $s \operatorname{diam}_{k}(G) \leq s \operatorname{diam}_{k}(H)$.

Li et al. [18] generalized the concept of Wiener index by Steiner distance. The Steiner Wiener $k$-index or k-center Steiner Wiener index $S W_{k}(G)$ of $G$ is defined by

$$
S W_{k}(G)=\sum_{\substack{S \subseteq V(G) \\|S|=k}} d_{G}(S) .
$$

For $k=2$, the above defined Steiner Wiener index coincides with the ordinary Wiener index. It is usual to consider $S W_{k}$ for $2 \leq k \leq n-1$, but the above definition 
implies $S W_{1}(G)=0$ and $S W_{n}(G)=n-1$. We refer to [18,19,21-24] for more details on Steiner Wiener index.

Furtula et al. [11] introduced the concept of Steiner Harary index. The Steiner Harary $k$-index or $k$-center Steiner Harary index $S H_{k}(G)$ of $G$ is defined as

$$
S H_{k}(G)=\sum_{\substack{S \subseteq V(G) \\|S|=k}} \frac{1}{d_{G}(S)} .
$$

For $k=2$, the above defined Steiner Harary index coincides with the ordinary Harary index. It is usual to consider $S H_{k}$ for $2 \leq k \leq n-1$, but the above definition implies $S H_{1}(G)=0$ and $S H_{n}(G)=\frac{1}{n-1}$.

In Section 2, we obtain the exact values of the Steiner Harary $k$-index of the path, complete graph, and complete bipartite graph. In Section 3, we obtain sharp lower and upper bounds for $S H_{k}$ for connected graphs and for trees. In Section 4 we establish some relations for $S H_{k}$ of trees. Our basic idea is from [18, 19].

\section{Results for Some Special Graphs}

Beginning this section, we note that the special case for $k=2$ of all formulas derived here for the Steiner Harary index, thus pertaining to the ordinary Harary index, are well known and mentioned many times in the earlier literature.

Proposition 2.1. Let $K_{n}$ be the complete graph of order $n$, and let $k$ be an integer such that $2 \leq k \leq n$. Then $S H_{k}\left(K_{n}\right)=\frac{1}{k-1}\left(\begin{array}{l}n \\ k\end{array}\right)$.

Proof. For any $S \subseteq V\left(K_{n}\right)$ and $|S|=k$, without loss of generality, we let $S=$ $\left\{u_{1}, u_{2}, \ldots, u_{k}\right\}$. Since $K_{n}$ is the complete graph of order $n$, it follows that the tree $T$ induced by the edges in $\left\{u_{1} u_{2}, u_{1} u_{3}, \ldots, u_{1} u_{k}\right\}$ is an $S$-Steiner tree, and hence $d_{K_{n}}(S) \leq k-1$. Since $|S|=k$, it follows that $d_{K_{n}}(S) \geq k-1$. Therefore, $d_{K_{n}}(S)=$ $k-1$. From the arbitrariness of $S$ and the symmetry of $K_{n}$, we have

$$
S H_{k}\left(K_{n}\right)=\sum_{\substack{S \subseteq V\left(K_{n}\right) \\
|S|=k}} \frac{1}{d_{K_{n}}(S)}=\frac{1}{k-1}\left(\begin{array}{l}
n \\
k
\end{array}\right),
$$

as desired.

Proposition 2.2. Let $K_{a, b}$ be the complete bipartite graph of order $a+b(1 \leq a \leq b)$, and let $k$ be an integer such that $2 \leq k \leq a+b$. Then

$$
S H_{k}\left(K_{a, b}\right)= \begin{cases}\frac{1}{k-1}\left(\begin{array}{c}
a+b \\
k
\end{array}\right)-\frac{1}{k(k-1)}\left(\begin{array}{l}
a \\
k
\end{array}\right)-\frac{1}{k(k-1)}\left(\begin{array}{l}
b \\
k
\end{array}\right), & \text { if } 1 \leq k \leq a ; \\
\frac{1}{k-1}\left(\begin{array}{c}
a+b \\
k
\end{array}\right)-\frac{1}{k(k-1)}\left(\begin{array}{l}
b \\
k
\end{array}\right), & \text { if } a<k \leq b ; \\
\frac{1}{k-1}\left(\begin{array}{c}
a+b \\
k
\end{array}\right), & \text { if } b<k \leq a+b .\end{cases}
$$

Proof. Let $G=K_{a, b}$, and let $U=\left\{u_{1}, u_{2}, \ldots, u_{a}\right\}$ and $W=\left\{w_{1}, w_{2}, \ldots, w_{b}\right\}$ be the two parts of $G=K_{a, b}$. 
First, we consider the case $1 \leq k \leq a$. For any $S \subseteq V(G)$ and $|S|=k$, we have $S \cap U=\varnothing$, or $S \cap W=\varnothing$, or $S \cap U \neq \varnothing$ and $S \cap W \neq \varnothing$. If $S \cap U=\varnothing$, then $S \subseteq W$. Without loss of generality, let $S=\left\{w_{1}, w_{2}, \ldots, w_{k}\right\}$. Then the tree $T$ induced by the edges in $\left\{u_{1} w_{1}, u_{1} w_{2}, \ldots, u_{1} w_{k}\right\}$ is an $S$-Steiner tree, and hence $d_{G}(S) \leq k$. Since $G=K_{a, b}$ is a complete bipartite graph, it follows that any tree connecting $S$ must use at least $k$ edges, and hence $d_{G}(S) \geq k$. Therefore, $d_{G}(S)=k$. If $S \cap W=\varnothing$, then $S \subseteq U$. Without loss of generality, let $S=\left\{u_{1}, u_{2}, \ldots, u_{k}\right\}$. Then the tree $T$ induced by the edges in $\left\{w_{1} u_{1}, w_{1} w_{2}, \ldots, w_{1} u_{k}\right\}$ is a Steiner tree connecting $S$, and hence $d_{G}(S) \leq k$. Since $G=K_{a, b}$ is a complete bipartite graph, it follows that any tree connecting $S$ must use at least $k$ edges, and hence $d_{G}(S) \geq k$. Therefore, $d_{G}(S)=k$. Suppose $S \cap U \neq \varnothing$ and $S \cap W \neq \varnothing$. Without loss of generality, let $S=\left\{u_{1}, u_{2}, \ldots, u_{x}, w_{1}, w_{2}, \ldots, w_{k-x}\right\}$. Then the tree $T$ induced by the edges in $\left\{u_{1} w_{1}, w_{1} u_{2}, w_{1} u_{3}, \ldots, w_{1} u_{x}, u_{1} w_{2}, u_{1} w_{3}, \ldots, u_{1} w_{k-x}\right\}$ is an $S$-Steiner tree, and hence $d_{G}(S) \leq k-1$. Since $|S|=k$, it follows that any tree connecting $S$ must use at least $k-1$ edges, and hence $d_{G}(S)=k-1$. Thus,

$$
\begin{aligned}
S H_{k}(G) & =\sum_{\substack{S \subseteq V^{(G)} \\
S \cap U=\varnothing}} \frac{1}{d_{G}(S)}+\sum_{\substack{S \subseteq V(G) \\
S \cap U=\varnothing}} \frac{1}{d_{G}(S)}+\sum_{\substack{S \subseteq V(G) \\
S \cap U \neq \varnothing, S \cap U \neq \varnothing}} \frac{1}{d_{G}(S)} \\
& =\frac{1}{k}\left(\begin{array}{l}
a \\
k
\end{array}\right)+\frac{1}{k}\left(\begin{array}{l}
b \\
k
\end{array}\right)+\frac{1}{k-1}\left[\sum_{\substack{a \\
x=1}}\left(\begin{array}{l}
a \\
x
\end{array}\right)\left(\begin{array}{c}
b \\
k-x
\end{array}\right)\right] \\
& =\frac{1}{k}\left(\begin{array}{l}
a \\
k
\end{array}\right)+\frac{1}{k}\left(\begin{array}{l}
b \\
k
\end{array}\right)+\frac{1}{k-1}\left[\left(\begin{array}{c}
a+b \\
k
\end{array}\right)-\left(\begin{array}{l}
b \\
k
\end{array}\right)-\left(\begin{array}{l}
a \\
k
\end{array}\right)\right] \\
& =\frac{1}{k-1}\left(\begin{array}{c}
a+b \\
k
\end{array}\right)-\frac{1}{k(k-1)}\left(\begin{array}{l}
a \\
k
\end{array}\right)-\frac{1}{k(k-1)}\left(\begin{array}{l}
b \\
k
\end{array}\right) .
\end{aligned}
$$

Next, we consider the case $a<k \leq b$. For any $S \subseteq V(G)$ and $|S|=k$, we have $S \cap U=\varnothing$ or $S \cap U \neq \varnothing$. If $S \cap U=\varnothing$, then $S \subseteq W$ and $d_{G}(S)=k$. Suppose $S \cap U \neq \varnothing$. Then $d_{G}(S)=k-1$, and hence

$$
\begin{aligned}
& S H_{k}(G)=\sum_{\substack{S \subseteq V(G) \\
S \cap U=\varnothing}} \frac{1}{d_{G}(S)}+\sum_{\substack{S \subseteq V(G) \\
S \cap U \neq \varnothing}} \frac{1}{d_{G}(S)} \\
& =\frac{1}{k}\left(\begin{array}{l}
b \\
k
\end{array}\right)+\frac{1}{k-1}\left[\sum_{x=1}^{a}\left(\begin{array}{l}
a \\
x
\end{array}\right)\left(\begin{array}{c}
b \\
k-x
\end{array}\right)\right] \\
& =\frac{1}{k}\left(\begin{array}{l}
b \\
k
\end{array}\right)+\frac{1}{k-1}\left[\sum_{x=1}^{\infty}\left(\begin{array}{l}
a \\
x
\end{array}\right)\left(\begin{array}{c}
b \\
k-x
\end{array}\right)\right] \\
& =\frac{1}{k}\left(\begin{array}{l}
b \\
k
\end{array}\right)+\frac{1}{k-1}\left[\left(\begin{array}{c}
a+b \\
k
\end{array}\right)-\left(\begin{array}{l}
b \\
k
\end{array}\right)\right] \\
& =\frac{1}{k-1}\left(\begin{array}{c}
a+b \\
k
\end{array}\right)-\frac{1}{k(k-1)}\left(\begin{array}{l}
b \\
k
\end{array}\right) \text {. }
\end{aligned}
$$


In this end, we consider the remaining case $b<k \leq a+b$. For any $S \subseteq V(G)$ and $|S|=k$, we have $S \cap U \neq \varnothing$ and $S \cap U \neq \varnothing$. Then $d_{G}(S)=k-1$, and hence

$$
S H_{k}(G)=\sum_{\substack{S \subseteq V(G) \\
S \cap U=\varnothing}} \frac{1}{d_{G}(S)}=\frac{1}{k-1}\left(\begin{array}{c}
a+b \\
k
\end{array}\right) .
$$

The proof is now complete.

From the above proposition, we can derive the following corollary.

Corollary 2.1. Let $S_{n}$ be the star of order $n(n \geq 3)$, and let $k$ be an integer such that $2 \leq k \leq n$. Then

$$
S H_{k}\left(S_{n}\right)=\frac{k n-n+k}{k^{2}(k-1)}\left(\begin{array}{l}
n-1 \\
k-1
\end{array}\right) .
$$

Proof. From Proposition 2.2, we have that $S H_{k}\left(S_{n}\right)=S H_{k}\left(K_{1, n-1}\right)=\left(\begin{array}{l}n \\ n\end{array}\right) \frac{1}{n-1}=\frac{1}{n-1}$ for $k=n$ and $S H_{k}\left(S_{n}\right)=S H_{k}\left(K_{1, n-1}\right)=\frac{1}{k}\left(\begin{array}{c}n-1 \\ k\end{array}\right)+\frac{1}{k-1}\left(\begin{array}{c}n-1 \\ k-1\end{array}\right)$ for $2 \leq k \leq n-1$. We conclude that

$$
S H_{k}\left(S_{n}\right)=\frac{1}{k}\left(\begin{array}{c}
n-1 \\
k
\end{array}\right)+\frac{1}{k-1}\left(\begin{array}{l}
n-1 \\
k-1
\end{array}\right)=\frac{k n-n+k}{k^{2}(k-1)}\left(\begin{array}{l}
n-1 \\
k-1
\end{array}\right) .
$$

Proposition 2.3. Let $P_{n}$ be the path of order $n(n \geq 3)$, and let $k$ be an integer such that $2 \leq k \leq n$. Then

$$
S H_{k}\left(P_{n}\right)=n \sum_{k-1 \leq t \leq n-1} \frac{1}{t}\left(\begin{array}{l}
t-1 \\
k-2
\end{array}\right)-\left(\begin{array}{l}
n-1 \\
k-1
\end{array}\right) .
$$

Proof. Let $V\left(P_{n}\right)=\left\{u_{1}, u_{2}, \ldots, u_{n}\right\}$. Choose $S \subseteq V\left(P_{n}\right)$ and $|S|=k$. Without loss of generality, let $S=\left\{u_{i_{1}}, u_{i_{2}}, \ldots, u_{i_{k}}\right\}$ where $i_{1} \leq i_{2} \leq \cdots \leq u_{i_{k}}$. Clearly, $k-1 \leq$ $d(S) \leq n-1$. Observe that $d(S)=d_{P_{n}}\left(u_{i_{1}}, u_{i_{k}}\right)$. Then $k-1 \leq d_{P_{n}}\left(u_{i_{1}}, u_{i_{k}}\right) \leq n-1$. Let $d_{P_{n}}\left(u_{i_{1}}, u_{i_{k}}\right)=t$. Thus, $k-1 \leq t \leq n-1$ and $1 \leq i_{1} \leq n-t$. Therefore, we have $(n-t)$ ways to choose $u_{i_{1}}$. Once the vertex $u_{i_{1}}$ is chosen, then $u_{i_{k}}=u_{i_{1}+t}$ is determined. Since $d_{P_{n}}\left(u_{i_{1}}, u_{i_{k}}\right)=t$, we have $\left(\begin{array}{c}t-1 \\ k-2\end{array}\right)$ ways to choose $u_{i_{2}}, u_{i_{3}}, \ldots, u_{i_{k}-1}$. So there are $(n-t)\left(\begin{array}{l}t-1 \\ k-2\end{array}\right)$ ways to determine $S$. Thus,

$$
\begin{aligned}
S H_{k}\left(P_{n}\right) & =\sum_{k-1 \leq t \leq n-1}(n-t) \frac{1}{t}\left(\begin{array}{l}
t-1 \\
k-2
\end{array}\right) \\
& =n \sum_{k-1 \leq t \leq n-1} \frac{1}{t}\left(\begin{array}{l}
t-1 \\
k-2
\end{array}\right)-\sum_{k-1 \leq t \leq n-1}\left(\begin{array}{l}
t-1 \\
k-2
\end{array}\right) \\
& =n \sum_{k-1 \leq t \leq n-1} \frac{1}{t}\left(\begin{array}{l}
t-1 \\
k-2
\end{array}\right)-\left(\begin{array}{l}
n-1 \\
k-1
\end{array}\right),
\end{aligned}
$$

as desired. 


\section{Lower And Upper Bounds For General Graphs}

The following proposition is immediate.

Proposition 3.1. Let $G$ be a connected graph of order $n$, $e \in E(G)$, and let $k$ be an integer such that $2 \leq k \leq n$. Furthermore, let $F$ be the graph with vertex set $V(F)=V(G)$ and edge set $E(G) \backslash e$. Then

$$
S H_{k}(F) \leq S H_{k}(G) \text {. }
$$

This straightforwardly leads to the following theorem.

Proposition 3.2. Let $G$ be a connected graph of order $n$, and $T$ a spanning tree of $G$. Let $k$ be an integer such that $2 \leq k \leq n$. Then

$$
S H_{k}(T) \leq S H_{k}(G)
$$

with equality if and only if $G$ is a tree.

A lower bound for the Steiner Harary index of an arbitrary tree is given by the next theorem.

Theorem 3.1. Let $T$ be a tree of order $n$, and let $k$ be an integer such that $2 \leq k \leq n$. Then

$$
n \sum_{k-1 \leq t \leq n-1} \frac{1}{t}\left(\begin{array}{l}
t-1 \\
k-2
\end{array}\right)-\left(\begin{array}{l}
n-1 \\
k-1
\end{array}\right) \leq S H_{k}(T) \leq \frac{k n-n+k}{k^{2}(k-1)}\left(\begin{array}{l}
n-1 \\
k-1
\end{array}\right) .
$$

Moreover, among all trees of order $n$, the star $S_{n}$ maximizes the Steiner Harary $k$-index whereas the path $P_{n}$ minimizes the Steiner Harary $k$-index.

Proof. The validity of the second inequality is verified by induction on $n$. For $n=k$, we have $d_{T}(S)=k-1=n-1$ for $S \subseteq V(T)$, where $T$ is a tree of order $n$. Then $S H_{k}(T)=S H_{n}(T)=\frac{1}{n-1}=\frac{1}{d_{T}(S)}=\frac{n^{2}-n+n}{n^{2}(n-1)}\left(\begin{array}{c}n-1 \\ n-1\end{array}\right)$, as desired. Assume now that the second inequality holds for all trees of order $n$. Let $T$ be a tree on $n+1$ vertices and $v$ its pendent vertex. Let $u$ be the vertex adjacent to $v$ in $T$. Furthermore, let $T^{\prime}$ be the subtree of $T$ induced by $V(T) \backslash v$. Then the inequality holds for $T^{\prime}$. By the induction hypothesis,

$$
S H_{k}\left(T^{\prime}\right) \leq \frac{k n-n+k}{k^{2}(k-1)}\left(\begin{array}{l}
n-1 \\
k-1
\end{array}\right) .
$$

If $v \in S$ but $u \notin S$, then $d_{T}(S) \geq k$ for $S \subseteq V(T),|S|=k$. If both $v \in S$ and $u \in S$, then $d_{T}(S) \geq k-1$ for $S \subseteq V(T),|S|=k$. Therefore, we have

$$
\begin{aligned}
S H_{k}(T) & =S H_{k}\left(T^{\prime}\right)+\sum_{\substack{S \subseteq V(T),|S|=k \\
v \in S, u \notin S}} \frac{1}{d_{T}(S)}+\sum_{\substack{S \subseteq V(T),|S|=k \\
u, v \in S}} \frac{1}{d_{T}(S)} \\
& \leq \frac{k n-n+k}{k^{2}(k-1)}\left(\begin{array}{l}
n-1 \\
k-1
\end{array}\right)+\frac{1}{k}\left(\begin{array}{l}
n-1 \\
k-1
\end{array}\right)+\frac{1}{k-1}\left(\begin{array}{l}
n-1 \\
k-2
\end{array}\right)
\end{aligned}
$$




$$
=\frac{(k-1)(n+1)+k}{k^{2}(k-1)}\left(\begin{array}{c}
n \\
k-1
\end{array}\right) .
$$

The first inequality is also verified by induction on $n$. For $n=k$, we have $d_{T}(S)=$ $k-1=n-1$ for $S \subseteq V(T)$, where $T$ is a tree of order $n$. Then $S H_{k}(T)=S H_{n}(T)=$ $\frac{1}{d_{T}(S)}=\frac{1}{n-1}=n \cdot \frac{1}{n-1}\left(\begin{array}{c}n-2 \\ n-2\end{array}\right)-\left(\begin{array}{c}n-1 \\ n-1\end{array}\right)$, as desired. Assume now that the second inequality holds for all trees of order $n$. Let $T$ be a tree on $n+1$ vertices and $v$ its pendent vertex. Furthermore, let $T^{\prime}$ be the subtree of $T$ induced by $V(T) \backslash v$. Then the inequality holds for $T^{\prime}$ and we obtain

$$
S H_{k}(T)=S H_{k}\left(T^{\prime}\right)+\sum_{\substack{S \subseteq V(T),|S|=k \\ v \in S}} \frac{1}{d_{T}(S)} .
$$

By the induction hypothesis,

$$
S H_{k}\left(T^{\prime}\right) \geq n \sum_{k-1 \leq t \leq n-1} \frac{1}{t}\left(\begin{array}{l}
t-1 \\
k-2
\end{array}\right)-\left(\begin{array}{l}
n-1 \\
k-1
\end{array}\right) .
$$

Let $V\left(T^{\prime}\right)=\left\{v_{1}, v_{2}, \ldots, v_{n}\right\}$. For the vertex $v \in V(T)$, we can find a subtree $T_{1}$ such that $v \in V\left(T_{1}\right)$ and $d\left(V\left(T_{1}\right)\right)=k-1$. Without loss of generality, let $V\left(T_{1}\right)=$ $\left\{v, v_{1}, v_{2}, \ldots, v_{k-1}\right\}$. Clearly, there is only one subset of $V\left(T_{1}\right)$ containing $v$ and hence

$$
\sum_{\substack{S \subseteq V(T),|S|=k \\ v, v_{1}, v_{2}, \ldots, v_{k-1} \in S}} \frac{1}{d_{T}(S)}=\frac{1}{d\left(V\left(T_{1}\right)\right)}=\frac{1}{k-1}
$$

Pick up a vertex from $V(T) \backslash\left\{v, v_{1}, v_{2}, \ldots, v_{k-1}\right\}=\left\{v_{k}, v_{k+1}, \ldots, v_{n}\right\}$, say $v_{k}$, such that $v_{k}$ is adjacent to one element of $\left\{v_{1}, v_{2}, \ldots, v_{k-1}\right\}$. Clearly, the tree $T_{2}$ induced by the edges in $E\left(T_{1}\right) \cup\left\{v_{k} v_{j}\right\}$ is of order $k+1$, where $1 \leq j \leq k-1$ and $v_{k} v_{j} \in E(T)$. Then $d_{T}\left(V\left(T_{2}\right)\right)=d_{T}\left(\left\{v, v_{1}, v_{2}, \ldots, v_{k-1}, v_{k}\right\}\right)=k$. It is clear that there are at most $\left(\begin{array}{l}k-1 \\ k-2\end{array}\right)$ subsets of $V\left(T_{2}\right)$ containing both $v$ and $v_{k}$. For each such subset $S \subseteq V\left(T_{2}\right)$ with $|S|=k, d_{T}(S) \leq d_{T}\left(V\left(T_{2}\right)\right) \leq k$. Thus, we have

$$
\sum_{\substack{S \subseteq V(T),|S|=k \\
v, v_{k} \in S}} \frac{1}{d_{T}(S)} \geq\left(\begin{array}{l}
k-1 \\
k-2
\end{array}\right) \frac{1}{d_{T}\left(V\left(T_{2}\right)\right)} \geq \frac{1}{k}\left(\begin{array}{l}
k-1 \\
k-2
\end{array}\right) .
$$

Pick up a vertex from $V(T) \backslash\left\{v, v_{1}, v_{2}, \ldots, v_{k-1}, v_{k}\right\}=\left\{v_{k+1}, v_{k+2}, \ldots, v_{n}\right\}$, say $v_{k+1}$, such that $v_{k+1}$ is adjacent to one element of $\left\{v_{1}, v_{2}, \ldots, v_{k}\right\}$. Clearly, the tree $T_{3}$ induced by the edges in $E\left(T_{2}\right) \cup\left\{v_{k+1} v_{j}\right\}$ is of order $k+2$, where $1 \leq j \leq k$ and $v_{k+1} v_{j} \in E(T)$. Then $d_{T}\left(V\left(T_{3}\right)\right)=d_{T}\left(\left\{v, v_{1}, v_{2}, \ldots, v_{k+1}\right\}\right)=k+1$. Obviously, we can find $\left(\begin{array}{c}k \\ k-2\end{array}\right)$ subsets of $V\left(T_{3}\right)$ containing both $v$ and $v_{k+1}$. Thus,

$$
\sum_{\substack{S \subseteq V(T),|S|=k \\
v, v k+1}} \frac{1}{d_{T}(S)} \geq\left(\begin{array}{c}
k \\
k-2
\end{array}\right) \frac{1}{d_{T}\left(V\left(T_{3}\right)\right)} \geq \frac{1}{k+1}\left(\begin{array}{c}
k \\
k-2
\end{array}\right) .
$$


Continue the above procedure, we get

$$
\begin{aligned}
S H_{k}(T)= & S H_{k}\left(T^{\prime}\right)+\sum_{\substack{S \subseteq V(T),|S|=k \\
v \in S}} \frac{1}{d_{T}(S)} \\
\geq & S H_{k}\left(T^{\prime}\right)+\sum_{\substack{S \subseteq V(T),|S|=k \\
v, v_{1}, v_{2}, \ldots, v_{k-1} \in S}} \frac{1}{d_{T}(S)}+\sum_{\substack{S \subseteq V(T),|S|=k \\
v, v_{k} \in S}} \frac{1}{d_{T}(S)} \\
& +\sum_{\substack{S \subseteq V(T),|S|=k \\
v, v_{k+1} \in S}} \frac{1}{d_{T}(S)}+\cdots+\sum_{\substack{S \subseteq V(T),|S|=k \\
v, v_{n} \in S}} \frac{1}{d_{T}(S)} \\
\geq & S H_{k}\left(T^{\prime}\right)+\frac{1}{k-1}\left(\begin{array}{l}
k-2 \\
k-2
\end{array}\right)+\frac{1}{k}\left(\begin{array}{c}
k \\
k-2
\end{array}\right)+\cdots+\frac{1}{n}\left(\begin{array}{l}
n-1 \\
k-2
\end{array}\right) \\
= & n \sum_{k-1 \leq t \leq n-1} \frac{1}{t}\left(\begin{array}{l}
t-1 \\
k-2
\end{array}\right)-\left(\begin{array}{l}
n-1 \\
k-1
\end{array}\right)+\sum_{k-1 \leq t \leq n-1} \frac{1}{t}\left(\begin{array}{l}
t-1 \\
k-2
\end{array}\right)+\frac{1}{n}\left(\begin{array}{l}
n-1 \\
k-2
\end{array}\right) \\
= & (n+1) \sum_{k-1 \leq t \leq n-1} \begin{array}{l}
1 \\
t
\end{array}\left(\begin{array}{l}
t-1 \\
k-2
\end{array}\right)-\left(\begin{array}{l}
n-1 \\
k-1
\end{array}\right)+\frac{1}{n}\left(\begin{array}{l}
n-1 \\
k-2
\end{array}\right) \\
= & (n+1) \sum_{k-1 \leq t \leq n} \frac{1}{t}\left(\begin{array}{l}
t-1 \\
k-2
\end{array}\right)-\left(\begin{array}{l}
n-1 \\
k-2
\end{array}\right)-\left(\begin{array}{l}
n-1 \\
k-1
\end{array}\right) \\
= & (n+1) \sum_{k-1 \leq t \leq n} \frac{1}{t}\left(\begin{array}{l}
t-1 \\
k-2
\end{array}\right)-\left(\begin{array}{c}
n \\
k-1
\end{array}\right) .
\end{aligned}
$$

We recall that Theorem 3.1 provides a generalization of the much older results known for the Harary index, i.e., it yields this previous result by setting $k=2$.

Theorem 3.2. Let $G$ be a connected graph of order $n$, and let $k$ be an integer such that $2 \leq k \leq n$. Then

$$
n \sum_{k-1 \leq t \leq n-1} \frac{1}{t}\left(\begin{array}{l}
t-1 \\
k-2
\end{array}\right)-\left(\begin{array}{l}
n-1 \\
k-1
\end{array}\right) \leq S H_{k}(G) \leq \frac{1}{k-1}\left(\begin{array}{l}
n \\
k
\end{array}\right) .
$$

Moreover, the lower bound is sharp.

Proof. From Proposition 2.1, we have $S H_{k}\left(K_{n}\right)=\frac{1}{k-1}\left(\begin{array}{l}n \\ k\end{array}\right)$. According to Proposition 3.1, each subgraph $G$ of $K_{n}$ with $E(G) \subseteq E\left(K_{n}\right)$ has Steiner Harary index less than the Steiner Harary of $K_{n}$. Since each graph of order $n$ is a subgraph of the complete graph, the inequality holds. From Proposition 3.2, we know that $S H_{k}(T) \leq S H_{k}(G)$, where $T$ is a spanning tree of $G$. Combining this with Theorem 3.1, we have $S H_{k}(G) \geq$ $S H_{k}(T) \geq S H_{k}\left(P_{n}\right)=n \sum_{k-1 \leq t \leq n-1} \frac{1}{t}\left(\begin{array}{c}t-1 \\ k-2\end{array}\right)-\left(\begin{array}{l}n-1 \\ k-1\end{array}\right)$, as desired.

\section{On Steiner Harary Indices of Trees}

For $k=n, n-1$, we have the following results for trees. 
Theorem 4.1. Let $T$ be a tree of order $n$, possessing $p$ pendent vertices. Then

$$
S H_{n-1}(T)=\frac{n^{2}-2 n-p}{(n-1)(n-2)},
$$

irrespective of any other structural detail of $T$.

Proof. Since $k=n-1$, the respective subsets $S$ contain all except one vertices of $T$. If the vertex missing from $S$ is pendent, then the vertices contained in $S$ form a tree of order $n-1$. Therefore $d_{G}(S)=n-2$. There are $p$ such subsets, contributing to $S H_{n-1}$ by $p \times \frac{1}{n-2}$.

If the vertex of $T$, not present in $S$, is non-pendent, then the vertices contained in $S$ cannot form a tree, and the respective Steiner tree must contain all the $n$ vertices of $T$. Therefore, $d_{G}(S)=n-1$. There are $n-p$ such subsets, contributing to $S H_{n-1}$ by $(n-p) \times \frac{1}{n-1}$.

Thus, $S H_{n-1}(T)=\frac{p}{n-2}+\frac{n-p}{n-1}$, which straightforwardly leads to (4.1).

Theorem 4.2. Let $T$ be a tree of order $n$, possessing $p$ pendent vertices. Let $q$ be the number of vertices of degree 2 in $T$ such that each of them is adjacent to a pendant vertex. Then

$$
S H_{n-2}(T)=\left[\left(\begin{array}{l}
p \\
2
\end{array}\right)+q\right] \frac{1}{n-3}+\left(\begin{array}{c}
n-p \\
2
\end{array}\right) \frac{1}{n-1}+\frac{p n-p^{2}-q}{n-2} .
$$

Proof. For any $S \subseteq V(G)$ and $|S|=n-2$, we let $\bar{S}=\{u, v\}$. If $d_{T}(u)=d_{T}(v)=1$, then $d_{T}(S)=n-3$ and this case contributes $S H_{n-2}$ by

$$
\sum_{\substack{u, v \in \bar{S} \\
d_{T}(u)=d_{T}(v)=1}} \frac{1}{d_{T}(S)}=\left(\begin{array}{l}
p \\
2
\end{array}\right) \frac{1}{n-3} .
$$

If $d_{T}(u) \geq 2$ and $d_{T}(v) \geq 2$, then $d_{T}(S)=n-1$ and this case contributes $S H_{n-2}$ by

$$
\sum_{\substack{u, v \in \bar{S} \\
d_{T}(u) \geq 2, d_{T}(v) \geq 2}} \frac{1}{d_{T}(S)}=\left(\begin{array}{c}
n-p \\
2
\end{array}\right) \frac{1}{n-1} .
$$

Suppose that $d_{T}(u)=1$ and $d_{T}(v) \geq 2$. If $d_{T}(u)=1, d_{T}(v)=2$ and $u v \in E(T)$, then $d_{T}(S)=n-3$. If $d_{T}(u)=1, d_{T}(v) \geq 3$ and $u v \in E(T)$, then $d_{T}(S)=n-2$. If $d_{T}(u)=1, d_{T}(v) \geq 2$ and $u v \notin E(T)$, then $d_{T}(S)=n-2$. Therefore, this case contributes $\mathrm{SH}_{n-2}$ by

$$
\begin{aligned}
S H_{n-2}(T) & =\sum_{\substack{u, v \in \bar{S} \\
d_{T}(u)=1, d_{T}(v) \geq 2}} \frac{1}{d_{T}(S)} \\
& =\sum_{\substack{u, v \in \bar{S}, u v \in E(T) \\
d_{T}(u)=1, d_{T}(v)=2}} \frac{1}{d_{T}(S)}+\sum_{\substack{u, v \in \bar{S}, u v \in E(T) \\
d_{T}(u)=1, d_{T}(v) \geq 3}} \frac{1}{d_{T}(S)}+\sum_{\substack{u, v \in \bar{S}, u v \notin E(T) \\
d_{T}(u)=1, d_{T}(v) \geq 2}} \frac{1}{d_{T}(S)}
\end{aligned}
$$




$$
=\frac{q}{n-3}+\frac{p-q}{n-2}+\frac{p(n-p-1)}{n-2} .
$$

From the above argument, we have

$$
\begin{aligned}
S H_{n-2}(T) & =\left(\begin{array}{l}
p \\
2
\end{array}\right) \frac{1}{n-3}+\left(\begin{array}{c}
n-p \\
2
\end{array}\right) \frac{1}{n-1}+\frac{q}{n-3}+\frac{p-q}{n-2}+\frac{p(n-p-1)}{n-2} \\
& =\left[\left(\begin{array}{l}
p \\
2
\end{array}\right)+q\right] \frac{1}{n-3}+\left(\begin{array}{c}
n-p \\
2
\end{array}\right) \frac{1}{n-1}+\frac{p n-p^{2}-q}{n-2} .
\end{aligned}
$$

Acknowledgements. The author is grateful to the referees for valuable comments and suggestions. This work is supported by the National Science Foundation of China (Nos. 11601254, 11551001, 11661068, 11161037, and 11461054) and the Science Found of Qinghai Province (Nos. 2016-ZJ-948Q, 2014-ZJ-907, and 2014-ZJ-721).

\section{REFERENCES}

[1] P. Ali, P. Dankelmann and S. Mukwembi, Upper bounds on the Steiner diameter of a graph, Discrete Appl. Math. 160 (2012), 1845-1850.

[2] A. T. Balaban, The Harary index of a graph, MATCH Commun Math. Comput. Chem. 75 (2016), 243-245.

[3] J. A. Bondy and U. S. R. Murty, Graph Theory, Springer, 2008.

[4] F. Buckley and F. Harary, Distance in Graphs, Addison-Wesley, Redwood, 1990.

[5] J. Cáceresa, A. Márquezb and M. L. Puertasa, Steiner distance and convexity in graphs, Eur. J. Combin. 29 (2008),726-736.

[6] G. Chartrand, O. R. Oellermann, S. Tian and H. B. Zou, Steiner distance in graphs, Časopis Pest. Mat. 114 (1989), 399-410.

[7] P. Dankelmann and R. Entringer, Average distance, minimum degree, and spanning trees, J. Graph Theory 33 (2000) 1-13.

[8] P. Dankelmann, O. R. Oellermann and H. C. Swart, The average Steiner distance of a graph, J. Graph Theory 22 (1996) 15-22.

[9] A. Dobrynin, R. Entringer and I. Gutman, Wiener index of trees: theory and application, Acta Appl. Math. 66 (2001), 211-249.

[10] R. C. Entringer, D. E. Jackson and D. A. Snyder, Distance in graphs, Czech. Math. J. 26 (1976), 283-296.

[11] B. Furtula, I. Gutman and V. Katanić, Three-center Harary index and its applications, Iranian J. Math. Chem. 7(1) (2016), 61-68.

[12] M. R. Garey and D. S. Johnson, Computers and Intractability-A Guide to the Theory of NP-Completeness, Freeman, San Francisco, 1979, pp. 208-209.

[13] W. Goddard and O. R. Oellermann, Distance in Graphs, in: M. Dehmer (Ed.), Structural Analysis of Complex Networks, Birkhäuser, Dordrecht, 2011, pp. 49-72.

[14] I. Gutman, B. Furtula and X. Li, Multicenter Wiener indices and their applications, J. Serb. Chem. Soc. 80 (2015), 1009-1017.

[15] I. Gutman, S. Klavžar and B. Mohar (Eds.), Fifty years of the Wiener index, MATCH Commun Math. Comput. Chem. 35 (1997), 1-159.

[16] I. Gutman and O. E. Polansky, Mathematical Concepts in Organic Chemistry, Springer, Berlin, 1986.

[17] X. Li and Y. Fan, The connectivity and the Harary index of a graph, Discrete Appl. Math. 181 (2015), 167-173. 
[18] X. Li, Y. Mao and I. Gutman, The Steiner Wiener index of a graph, Discuss. Math. Graph Theory 36(2) (2016), 455-465.

[19] X. Li, Y. Mao and I. Gutman, Inverse problem on the Steiner Wiener index, Discuss. Math. Graph Theory, in press.

[20] B. Lučić, A. Miličević, S. Nikolić and N. Trinajstić, Harary index-twelve years later, Croat. Chem. Acta 75 (2002), 847-868.

[21] Y. Mao, Z. Wang and I. Gutman, Steiner Wiener index of graph products, Trans. Combin. 5(3) (2016), 39-50.

[22] Y. Mao, Z. Wang, I. Gutman and A. Klobučar, Steiner degree distance, MATCH Commun. Math. Comput. Chem. 78(1) (2017), 221-230.

[23] Y. Mao, Z. Wang, I. Gutman and H. Li, Nordhaus-Gaddum-type results for the Steiner Wiener index of graphs, Discrete Appl. Math. 219 (2017), 167-175.

[24] Y. Mao, Z. Wang, Y. Xiao and C. Ye, Steiner Wiener index and connectivity of graphs, Utilitas Math., in press.

[25] O. R. Oellermann and S. Tian, Steiner centers in graphs, J. Graph Theory 14 (1990), 585-597.

[26] D. H. Rouvray, Harry in the limelight: The life and times of Harry Wiener, in: D. H. Rouvray, R. B. King (Eds.), Topology in Chemistry - Discrete Mathematics of Molecules, Horwood, Chichester, 2002, pp. 1-15.

[27] D. H. Rouvray, The rich legacy of half century of the Wiener index, in: D. H. Rouvray, R. B. King (Eds.), Topology in Chemistry - Discrete Mathematics of Molecules, Horwood, Chichester, 2002, pp. 16-37.

[28] H. Wiener, Structural determination of paraffin boiling points, J. Am. Chem. Soc. 69 (1947), 17-20.

[29] K. Xu, K.C. Das and N. Trinajstić, The Harary Index of a Graph, Springer, Heidelberg, 2015.

[30] K. Xu, M. Liu, K. C. Das, I. Gutman and B. Furtula, A survey on graphs extremal with respect to distance-based topological indices, MATCH Commun. Math. Comput. Chem. 71 (2014), 461-508.

Department of Mathematics

Qinghai Normal University

Xining, Qinghai 810008

ChInA

E-mail address: maoyaping@ymail.com 